\title{
Personal Reminiscences about Professor Masaaki Sugihara
}

\section{Kazuo Murota ${ }^{1}$}

Published online: 26 August 2019

() The JJIAM Publishing Committee and Springer Japan KK, part of Springer Nature 2019

Professor Masaaki Sugihara passed away at the age of 64 on January 5, 2019. He made fundamental contributions to numerical analysis, working at the University of Tsukuba, Hitotsubashi University, Nagoya University, The University of Tokyo, and Aoyama Gakuin University. He was an area editor of the Japan Journal of Industrial and Applied Mathematics from 2006 to 2018. In 2008 he served as vice president of the Japan Society for Industrial and Applied Mathematics.

I met him for the first time beside a line printer in the computer room at The University of Tokyo. It was in 1976, when I was engaged in some homework and he was probably working on his bachelor's thesis. Both of us were students in the Department of Mathematical Engineering of the university. I was a third-year student and he was one year ahead of me.

In those days, computers received inputs (programs and data) via punch cards, and we had to wait a long time before we got any output from a line printer. We started to talk to each other while we were waiting for our outputs, without anticipating, of course, that we would continue our friendship for more than 40 years as students and co-authors of more than ten papers and six books.

As a graduate student, he belonged to a research group majoring in statistics. Without very strong motivation, I also decided to go on to the graduate program and chose the group that looked easiest to me. He was there, and I learned how to study from him. First of all, he told me that I should study more seriously. But his way of speaking was not demanding or preachy, but, rather, used kind and gentle words even with some exaggerated compliments. (I realized only later that this was always the case with his manner of speaking.) At any rate, I became interested in studying for myself, and then I looked at what he was doing. Instead of reading every detail of a single book, he read a couple of related books in parallel to reconstruct the results in his own notebook. I realized, although vaguely, that it was important to arrive at my own understanding by making up my own story in my notebook. Throughout the period of my master's

This remembrance is based on an article first published in Japanese in the JSIAM Online Magazine (K1902A, February 2019, http://jom.jsiam.org/?article=K1902A).

Kazuo Murota

murota@tmu.ac.jp

1 Tokyo Metropolitan University, Tokyo, Japan 
degree program, he gave me a lot of technical advice, and he kindly checked the messy calculations in my master's thesis. Sometimes we enjoyed competing with each other in our skill of asymptotic expansions.

While he was in the doctoral program, he worked on numerical methods of integration, by himself. First, he got interested in a number-theoretic method for multidimensional integration. This method makes use of the fact that the fractional parts of integer multiples of, say, $\sqrt{2}$ are uniformly distributed in the unit interval between 0 and 1, whereas many other numerical methods of integration are based on a weighted sum of function values evaluated at certain mesh points. I was surprised to see the tools used in his research, which evidenced his deep and broad knowledge in mathematics. This work was published as a paper titled "A note on Haselgrove's method for numerical integration" (Mathematics of Computation, 1982).

His interest in multi-dimensional integration developed into the method of good lattice points, and the results of his research were compiled in his doctoral thesis titled "Study of Quasi Monte-Carlo Methods" in 1982. With some additional results, this work was later published as "Method of good matrices for multi-dimensional numerical integrations-An extension of the method of good lattice points" (Journal of Computational and Applied Mathematics, 1987).

When he was finishing his doctoral thesis, he made a presentation about the method of good lattice points at an annual meeting of the Mathematical Society of Japan, and it happened that Professor Masatake Mori was in the audience. This was a most fortunate encounter for Sugihara, and also for the Japanese community of numerical computation. He soon was appointed assistant professor at the Institute of Electronics and Information Engineering of the University of Tsukuba in 1982. At that time, the institute was undoubtedly the focal point of numerical computation in Japan, at which Professor Mori as well as Professors Yasuhiko Ikebe, Ryo Natori, and Yoshio Oyanagi were faculty members. The next year, I fortuitously was appointed to a position at the same university, but in a different institute, which happened to be located in the same building as the numerical computation group. In this way, Professor Sugihara and I continued to meet almost everyday.

Around this time, his main interest shifted toward the DE formula, which is a highly efficient method of numerical integration devised by Professor Hidetosi Takahasi and Professor Mori in 1974. The name of this formula reflects its being based on a transformation of the variable such that the transformed integrand, defined over $(-\infty,+\infty)$, has a double exponential decay toward $\pm \infty$. Being equipped with mathematical beauty and practical effectiveness at the same time, the DE formula is one of the major Japanese contributions to applied mathematics.

The original paper by Takahasi and Mori had already given a theoretical error analysis for the DE formula, and in addition, discussed why the DE decay of the transformed integrand is optimal. Their argument was mathematically sound and proper, but it was also subjected to a critical question concerning its mathematical rigor. Indeed, at that time Professor Frank Stenger had already constructed a rigorous theory about the lower bound of the error realizable by any numerical integration formula. The theory showed that the single exponential (SE) formula should be the optimal formula, which apparently did not allow the DE formula to have a smaller error than the SE formula. This apparent inconsistency strongly motivated Professor Sugihara to work for a func- 
tional analytic foundation of the DE formula that would be eligible for international acceptance.

He spent ten years in solving this problem. After countless revisions of the theory he finally published a paper titled "Optimality of the double exponential formulaFunctional analysis approach" (Numerische Mathematik, 1997). The optimality of a numerical integration formula can be discussed only with respect to a class of integrand functions to which the integration formula is applied. Sugihara's paper discusses the optimality of the DE formula by identifying a class of integrand functions which is meaningful from practical applications and for which the optimality of the DE formula can be proved. The conclusion of the paper consists, roughly, of the following two statements.

1. For a certain class of integrand functions, we can provide an error estimate for the DE formula, i.e., an upper bound of the errors incurred by the DE formula when applied to functions belonging to that function class. Furthermore, we can give a lower bound of the errors for any integration formula when applied to functions belonging to that function class. This lower bound, which shows a limit on the possible performance of any integration formula, coincides essentially with the error estimate for the DE formula. Therefore, we may regard the DE formula as the optimal formula with respect to that class of integrand functions.

2. It is not possible to set up a natural function class for which the errors are smaller than that of the DE formula.

This was Sugihara's theory on the optimality of the DE formula. Proving the optimality of anything is always a difficult task, because it inevitably involves a proof of the nonexistence of better solutions. His contributions to numerical integration are described in Chapter 11 of his book Elements of Numerical Computation (Iwanamishoten, 1994, in Japanese).

For the ten years of his struggle with DE optimality, from the very beginning until the completion of his paper, I was a constant witness of the development. The DE optimality paper was "completed" many times, but was revised as many times to incorporate substantial improvements. As a result of such persistence and efforts the DE formula has achieved the "global standard" needed for international recognition.

After the DE optimality project, he proceeded to a method that combines the DE transformation and the Sinc approximation. With young colleagues and students, he developed various algorithms of the DE-Sinc method. The Japanese tradition of DE transformations, initiated by Professors Takahasi and Mori and developed by Professor Sugihara, is now being inherited by the next generation. I would like to continue to be a supporting observer of this tradition.

Another main research area of Professor Sugihara was linear computation (numerical computation for matrices). Among others, he was interested in conjugate gradient methods. His interest originated from an expository article by Professor Takahasi titled "The Lanczos principle and numerical analysis" (Mathematical Sciences, 1976, in Japanese). In this article a certain phenomenon in the orthogonalization of vectors is called the Lanczos principle and its effective use is demonstrated for numerical computations in several (seemingly unrelated) contexts. One of these examples revealed 
the underlying mechanism of the conjugate gradient method. Professor Sugihara and I were fascinated by this unifying view.

From our student days majoring in mathematical engineering, we were concerned with the issue of invariance in modeling and computation, which we had learned from Professor Masao Iri. We wondered how the issue of invariance is related to the preprocessing for the conjugate gradient methods, which was just emerging as an important practical technique. Thus we arrived at a problem that seemed interesting to us: How should we understand the preprocessed conjugate gradient methods in the light of the Lanczos principle and tensorial invariance? It took more than a year for us to reach a definite conclusion. Although this "result" of our discussion was never published as a paper, what we thought and discussed during this period guided us in subsequent research. His views on linear computation are compiled in his book Theoretical Numerical Linear Algebra (Iwanami-shoten, 2009, in Japanese).

While he constructed a very rigid theoretical framework around the DE transformation, his scientific mind was, in fact, broad and flexible. With his balance between theory and practice, he enjoyed collaboration with many people in many different areas in mathematical engineering. He was also good at supervising students. Not only did he choose appropriate research topics for students, but he guided them according to their personality and capability. Students who got lost in life were encouraged by his smile and found their ways to go on further.

Looking back, I deeply appreciate my good fortune that I have had a close friend with whom I studied and worked together. From the bottom of my heart, I pray that his soul may rest in peace.

\section{Brief CV of Professor Masaaki Sugihara}

Date of birth: December 22, 1954

[Education]

- March 1973: Graduated from Gifu Prefectural Gifu High School

- March 1977: Graduated from Department of Mathematical Engineering and Instrumentation Physics, The University of Tokyo

- March 1979: Graduated from Master's Program of Mathematical Engineering and Instrumentation Physics, The University of Tokyo

- March 1982: Graduated from Doctoral Program of Mathematical Engineering and Instrumentation Physics, The University of Tokyo

[Degree] Doctor of Engineering (March 1982)

[Career]

- April 1982: Assistant Professor, Faculty of Engineering, The University of Tokyo

- April 1982: Assistant Professor, Institute of Electronics and Information Engineering, University of Tsukuba

- January 1987: Lecturer, Institute of Electronics and Information Engineering, University of Tsukuba

- April 1987: Lecturer, Faculty of Economics, Hitotsubashi University

- April 1988: Associate Professor, Faculty of Economics, Hitotsubashi University 
- April 1991: Associate Professor, Faculty of Engineering, The University of Tokyo

- February 1997: Professor, Faculty of Engineering, Nagoya University

- February 2005: Professor, Graduate School of Information Science and Technology, The University of Tokyo

- April 2013: Professor, College of Science and Engineering, Aoyama Gakuin University

- December 2014: Professor Emeritus, Nagoya University

- June 2015: Professor Emeritus, The University of Tokyo

[The Japan Society for Industrial and Applied Mathematics]

Fellow, Vice President (2008), Outstanding Achievement Award (2014), Area editor of Japan Journal of Industrial and Applied Mathematics (2006 - 2018)

Publisher's Note Springer Nature remains neutral with regard to jurisdictional claims in published maps and institutional affiliations. 